\title{
Students Meeting Advanced Mathematics for the First Time: Can Mathematics Education Research Help?
}

\author{
MARIA MEEHAN
}

\begin{abstract}
In this article, a brief survey is presented of some results from the mathematics education research literature, relating to difficulties students have when meeting advanced mathematics for the first time.
\end{abstract}

\section{INTRODUCTION}

In the spring semester 2002, I was assigned to teach a course called Introduction to Analysis to a class of 32 second year Arts students, who were taking Mathematical Studies as one of their degree subjects. Mathematical Studies is a three year degree course in UCD, and any student who achieves at least $50 \%$ as their final overall mark will graduate with an honours degree.

Introduction to Analysis is taught over twelve weeks, with three one hour lectures and one one hour tutorial each week. Topics such as the real numbers, sequences, series and power series are covered. The students taking the course would already have taken a first year course in Calculus, and a one semester, second year course in Calculus of Several Variables.

In the fourth week of the course, I introduced the class to the definition of a convergent sequence of real numbers. Aware that there would probably be cognitive difficulties with this concept, I spent quite a lot of time on it. We looked at the sequence $\left(a_{n}\right)$ where $a_{n}=1 / \sqrt{n}$. We wondered about how "far out in the sequence" we would have to go until we reached a term $a_{N}$, so that this term and each one after it would lie within $1 / 10$ of zero. Then we changed the $1 / 10$ to $1 / 50$ and wondered about how this would change our

Summary of a talk on the same subject delivered at the Annual Meeting of the IMS in Cork in September 2002. 
answer. Then we replaced the $1 / 50$ with $\epsilon$ where $\epsilon>0$, and tried to answer the question in general.

We looked at many other examples and proved some of the algebraic limit properties. We discussed bounded sequences, monotone sequences, subsequences and Cauchy sequences. The Monotone Convergence Theorem and the Bolzano-Weierstrass Theorem were proved and the connection between Cauchy sequences and convergent sequences was studied in detail. This material was covered in approximately 12 lectures and to emphasise the importance of the definition of a convergent sequence, I usually started each of these lectures by writing the definition on the board.

However, in a class test a few weeks later, when asked to state the definition of a convergent sequence of real numbers, less than one-third of the class stated it correctly! These students had been working hard, had been participating in lectures and tutorials and had given no indication that they didn't understand this concept. In fact, it seemed that they felt they did understand. Each student, apart from one, reproduced something that looked like the definition $\left(\epsilon, N\right.$, "for every", "there exists" and " $\left|a_{n}-l\right|<\epsilon$ " appeared in various combinations). Also, when asked for examples of sequences which are, or are not, convergent, bounded or monotone, most of the students who couldn't state the definitions correctly, could still present examples. Similarly, when given true or false statements concerning combinations of these concepts, many could choose the correct answer and provide counterexamples where appropriate. If a proof was required, it seemed that a number of students were relying on memory (if they had seen the result before) to reproduce it. A few more relied on "proof by example".

However tempting it might have been to assign blame to the students, I didn't really believe that the class was at fault. I didn't believe that the blame lay entirely at my feet either. It just seemed that the class and myself were thinking very differently about the subject. A lot of pedagogical questions occurred to me, but the one which I really wanted some insight to was:

Why do students seem to regard definitions as irrelevant?

Aware of the emerging research in the area of third level mathematics education, I wondered if the answer to my question lay here.

Research on mathematics education at the undergraduate level is in its infancy. Most of the major articles in the area have been 
written in the 1990s, with just a few before that [7]. Can this new research help the third level lecturer in mathematics? The lecturer who turns to research in mathematics education expecting to be told the correct way to teach a class or a particular topic will be disappointed [7]. There are no proofs and no definitive answers, but one of the things that research in this area can do, is give the lecturer some insight into the difficulties that the student encounters. I needed some light shed on how students react to definitions in advanced mathematics, and despite being a relatively new field, I was delighted to find that some excellent studies have been undertaken on this very topic.

\section{Concept Image and Concept Definition}

Perhaps the most well known study relating to definitions in advanced mathematics, is the paper by David Tall and Shlomo Vinner [10]. In this paper, the authors make the distinction between the formal definition of a mathematical concept, and the image that an individual has in his or her head relating to the concept. The term concept definition is used to refer to the mathematical definition of a concept. Moore [6] describes it as "a formal verbal definition that accurately explains the concept in a noncircular way". The term concept image is used to describe "the total cognitive structure that is associated with the concept, which includes all the mental pictures and associated properties and processes" [10]. Everything that a person knows about a concept, be it conscious or unconsious, correct or incorrect, is included in the concept image. Of course the concept definition may or may not be a part of the concept image.

Tall and Vinner point out that only a certain portion of the concept image may be evoked at any given time. They refer to this as the evoked concept image. They note that a concept image may contain conflicting pieces of information. The individual may remain unaware of this, unless the conflicting pieces are evoked simultaneously, in which case they become cognitive conflict factors. The conflict must then be resolved by the individual and the concept image adjusted. For instance, studies have shown [11] that many students believe incorrectly that a convergent sequence can "never reach" its limit. This may be part of a person's concept image, while simultaneously the person may correctly believe that the sequence $\left(a_{n}\right)$, where $a_{n}=(-1)^{2 n}$, converges to 1 . If both of these facts are evoked simultaneously, cognitive conflict is likely to occur. 
Many of our third level students will have met concepts like functions, limits, differentiation and continuity informally in their secondary education. It is most likely that formal definitions of these concepts will not have been presented to the student. This is not a criticism of the curriculum. Indeed, most would agree that it is a positive thing that students meet these concepts informally before being exposed to the rigour of the formal definition. In fact, Burn [3] notes that most advanced mathematical theory is presented in textbooks and journal articles in the sequence definition, theorem, proof, corollary. He argues that the mathematician most likely came to the result in the reverse order:

The research mathematician may come to his results starting from some special cases, which will appear as corollaries in the final version, from which he gets the idea, which is worked with until he has a proof. Then the theorem is what has been proved. At this point, he formulates his definition so as to make the theorem and proof as neat as possible. (Burn, [3])

Therefore in many cases, it makes sense for our students to be familiar with certain concepts before they encounter the formal definition.

Tall and Vinner were interested in trying to study the concept images students had of certain mathematical concepts. Vinner [11] notes that if you want to find out if a person knows a particular concept definition, you simply ask them to state it. However to study a person's concept image, the researcher must ask indirect questions which will expose certain parts of the image. Several studies are described in $[10,11]$ which attempt to investigate how individual concept images differ from the formal theory. The authors attempt to study the concept images that students have of functions, continuous functions, limits of functions, limits of sequences and tangents to a graph of a differentiable function. I will very briefly mention some of the results here. (The interested reader will find excellent descriptions of the results in $[10,11]$.)

As mentioned above, Tall and Vinner found that many students believe that a convergent sequence "must not reach its limit" and is necessarily monotonic. In relation to continuous functions, a popular concept image was that a continuous function must be given by one 
formula only. For example a function such as

$$
f(x)= \begin{cases}0, & x<0 \\ x^{2}, & x \geq 0\end{cases}
$$

would not be considered continuous. In [11], a study is described where 278 first year university students were presented with graphs of three functions: $g(x)=x^{3}, h(x)=\sqrt{|x|}$ and the function $f$ above. They were asked to state whether they thought it was possible to draw a tangent to each of these graphs at the point $(0,0)$. If, in their opinion it was possible, they were then asked to specify how many tangents there were (one, two, three, infinitely many) and to sketch them. The notions that a tangent can "only cut the curve at one point" and that a tangent "can't intersect the curve" were prevalent. The tangent had been defined in class as either the limit of secants or as a line having a common point with the function graph whose slope is the derivative at this particular point. $41 \%$ of students gave one of these definitions when asked, whereas $35 \%$ gave descriptions that suit the tangent to a circle. The concept image of tangent to a circle was so strong for some students that the actual definition of tangent to a curve seemed superfluous.

\section{Concept Image Formation Using the Concept DEFINITION}

In the studies in $[10,11]$, the students had informally met a lot of the concepts before being introduced to the formal definition. In [4], Randall Dahlberg and David Housman attempted to study how students initially develop an understanding of a formal concept. In other words, when presented with a new concept definition for the first time, they essentially wanted to observe how the concept image was formed (if at all). 11 students from Housman's university took part in the study. Of these 10 were seniors and one was a junior, and 10 were mathematics majors, with one being a computer science major. Based on their previous mathematical performance the students represented four ability groups.

Each student was interviewed individually, and throughout the interview the student was encouraged to state or write whatever he or she was thinking. The interviewer could then attempt to monitor when a learning event had occured, that is, "when the student communicates and applies a new understanding of the concept" [4]. 
At the start of the interview, the student was presented with the definition page. On this page was the following definition:

A function is called fine if it has a root (zero) at each integer.

The authors found that four basic learning strategies emerged: example generation, reformulation, decomposition and synthesis, and memorisation. Many of the better students used example generation - they tried to find examples of fine functions. Graphs of the constant zero function and a sinusoidal graph, which crossed the $x$-axis at each integer, were some of the examples given. Three students restated the definition, with a reformulation such as $f(n)=0$, for all $n \in \mathbf{Z}$ being given. Some of the weaker students "broke down" the definition, tried to examine each of the separate parts, and then put the meaning together at the end. Finally, some students wrote nothing down and when prompted, said they usually just memorised definitions without really understanding them.

At this point in the interview the students were presented with a generation page which encouraged them to give an example of a function which is fine, one which is not, and also to state in their own words what a fine function is. Essentially, the student was being encouraged to generate examples and reformulate. Dahlberg and Housman found that those who employed example generation initially, switched to reformulation and vice versa, with additional learning events occuring. Most of those who had initially employed one of the other two learning strategies didn't seem to experience any further learning events.

In the remainder of the interview, each student was given a verification page and conjecture page. The verification page contained examples of functions, and the student had to decide which of these were fine. Statements about fine functions appeared on the conjecture page, and the student had to determine the validity of each of these. Overall the authors found that example generation played a significant role in the development of the concept image.

Students who consistently employed example generation had more learning events, were able to encapsulate more examples into their concept image of fine function, and were more able to use these examples than those who primarily used other learning strategies. (Dahlberg and Housman, [4]) 


\section{Example Generation}

Clearly the more examples one has of a concept, the better informed the concept image will be. If a person has many (correct) examples of a concept in his or her concept image, then it seems likely that the concept image will be more in line with the formal theory. As mathematicians we know that examples play an important role in mathematical research. Very often ideas come from observing and working with examples of various concepts. In addition, when introduced to a new concept or theory we often try to find examples which will aid our understanding. We should therefore try to emphasise the importance of examples to our students.

Frequently, lecturers and textbooks when presenting a new topic, give a few "well-worn" examples of a concept and leave it at that. Do students interpret these as being more "correct" examples than those which they generate themselves? Do they try to generate their own? Are we denying them the opportunity to expand their concept images by not encouraging them to generate their own? Do they find it difficult to generate examples?

In [5] Orit Hazzan and Rina Zazkis analyse the approaches that students take, and the difficulties they encounter, when asked to give an example of a mathematical entity. The following are two of the tasks they presented to students:

(A) Give an example of a 6-digit number divisible by 17 .

(B) Give an example of a function whose value at $x=3$ is -2 .

They note that such tasks could be thought of as "inverses" of those students are normally asked to do: show that 163,710 is divisble by 17 ; show that $f(3)=-2$ if $f(x)=2 x-8$.

Several approaches were observed. Random trial and error was a common one. For example with (A), several students randomly picked 6 -digit numbers and checked if they were divisble by 17 . Another approach related to this is informed trial and error. For example on finding that a random 6-digit number has remainder 2 when divided by 17, the student subtracted 2 from the random number to obtain the example. It was also noted that a common approach was to design an algorithm. When asked to give 5 additional examples of functions that satisfied (B), the authors observed that students were anxious to find an algorithm to produce the examples. 
Hazzan and Zazkis also observed that very few students gave "trivial" examples. For example, 170,000 is an example of (A). The authors note that this may be because the student feels that a 6 digit number should contain a variety of digits or that the lecturer is expecting something more. Another observation was that students were anxious to check their answers. For example, if 17 was multiplied by 9,630 to obtain 163,710 , the tendency was then to check the divisibility of this number by 17 . Finally, the authors note that the fact that there is no unique example of $(\mathrm{A})$ or $(\mathrm{B})$ caused some discomfort. This may be that students have been conditioned to expect one correct answer to a problem. Thus while generating examples may be second nature to the mathematician, it can be a difficult task for students and one worth engaging them in.

\section{From Definition to Proof}

On completing a course such as the Introduction to Analysis mentioned in Section 1, we generally expect that the successful student will not only be familiar with certain concepts and their definitions, but will also be able to reason using definitions. As lecturers in advanced mathematics, we expect our students to be able to present proofs relating certain concepts. Even with a well developed concept image, this can prove difficult for the student.

Lara Alcock and Adrian Simpson describe in [1] some responses of Real Analysis students from the University of Warwick, when faced with proving that a convergent sequence $\left(a_{n}\right)$ is bounded. They first recount the response of a student, Wendy, who tries to "generalise from a prototype". When trying to prove the result, the student seems to be arguing from examples of monotonic sequences. The authors note that it is not clear whether she feels that all convergent sequences are monotonic, or whether she deems them more important than other convergent sequences. This may be because many of the examples she would have seen of convergent sequences, would have been monotonic.

Secondly they describe the approach of a student, Cary, who tries to "abstract properties from a prototype". Cary appreciates that not all convergent sequences are monotonic and seems to have a richer concept image of convergent sequence than Wendy. However, when trying to prove the result, it seems that he tries to extract the properties of convergent sequence from the examples in his concept 
image. It doesn't occur to him, until prompted, to use the definition. (Note that this is how the research mathematician might reach a conclusion, and the authors argue that this type of thinking is to be encouraged.)

Alcock and Simpson observe that Wendy's situation could improve if she had a better awareness of examples of convergent sequences. They argue that "such an awareness should make her less likely to overgeneralize from a restricted range of cases, and more likely to recognize the potential pitfalls of relying on relatively fixed images". Cary is in a better situation than Wendy, in that he has a good awareness of examples of convergent sequences, but still needs to make that final step and refer to the definition.

The authors suggest promoting the use of collaborative work in class. They note that the feedback, challenge and debate experienced in group work, may result in the individual student being more critical of his or her own work. This may help a student like Wendy to have a better idea of what objects belong to a given category. They also suggest reversing some of the usual tasks that students are given. Rather than ask the student to demonstrate that a given object belongs to a given category, ask him or her to provide examples of a given category. Students like Cary may then be better able to see the link between the definition and examples.

\section{Conclusions}

I return to the question posed in Section 1:

Why do students seem to regard definitions as irrelevant?

In everyday life we very rarely use formal definitions to understand the world around us. We know what is meant by "car", "shoes" and "cat" because we have seen enough examples of these concepts. Is it even possible to formally define these? In many instances in everyday life we work from "prototypical examples". If someone told me that they owned a cat, I would automatically think small and furry (pet cat), and not big and ferocious (wildcat). Thus in everyday life, one could say that we work and argue and make decisions from the prototypical examples we have in our heads.

In mathematics, concepts are precisely determined by their definitions. No one example of a concept is better than another. We know exactly what objects belong to a given category. We make our arguments based on definitions. Compared with the way we think 
in everyday life, one might conclude that thinking mathematically does not come naturally!

Think about a student taking an "everyday life" approach to the concept of convergent sequence. The student develops a concept image of convergent sequence which is likely to contain examples of convergent sequences. These examples will probably have come from the lecturer or textbook. In the "everyday life" approach, the definition may now be thrown away since the image is formed. Vinner [11] uses the "scaffolding metaphor": once the building is up, the scaffolding can be removed.

Vinner [11] claims that "one of the goals of teaching mathematics should be changing the thought habits from the everyday life mode to the technical mode", although he recognises the difficulty in this. One suggestion is that, if possible, the mathematical definition should be acquired in the everyday life mode by using examples to build up the concept image. The formal definition can then be presented (or perhaps if it is not too difficult, the student could be asked for his or her definition). Vinner notes that if the student is taking an advanced mathematics course, where ultimately he or she will have to use the definition in reasoning and proofs, one must do more than simply introduce the formal definition. The conflicts between the concept image and formal theorem need to be addressed and discussed in detail (see Section 2). He also suggests that the student must be given tasks which require the formal theory, and can't be answered by only consulting the concept image (this is one for the imaginative lecturer).

Throughout this survey of the literature we have seen the role played by examples in helping the student understand a concept. In Section 2, we saw how the concept image and formal theory can diverge. One could use the results (and examples) in $[10,11]$ to expose cognitive conflicts factors, which may help to realign the concept image and the formal theory. We noted in Section 3 that example generation is a habit that is worth encouraging in our students. This can prove difficult for the student as discussed in Section 4, but by "reversing" the tasks usually assigned and asking students to "give an example of ...", we can hopefully encourage this practice. In Section 5, many of these themes and suggestions appeared again.

In the business world there is an expression "analysis by paralysis". When I first read the papers discussed in this article, I was slightly overwhelmed at the task facing me as a lecturer. How could 
I possibly present a lecture in advanced mathematics which would encompass all the above suggestions and which would ensure that my students wouldn't make the mistakes or fall into the traps described above? Paralysis set in.

On reflection, I think it is impossible. However at least now I am more aware of the pitfalls and plan to make some changes in the order that I present the material, in the type of questions I ask, and I intend to encourage group work in class. And next semester, if one third of my second year Introduction to Analysis class correctly state the definition of a convergent sequence, I'll be over the moon!!

\section{REFERENCES}

[1] L. Alcock and A. Simpson, Two components in learning to reason using definitions, Proceedings of the 2nd International Conference on the Teaching of Mathematics (at the Undergraduate Level), 2002, CD-Rom.

[2] L. Alcock and A. Simpson, The Warwick analysis project: practice and theory, in D. Holton (ed.), The Teaching and Learning of Mathematics at University Level - An ICMI Study, Kluwer Acad. Publ., Dordrecht, 2001, 99-111.

[3] R. Burn, The genesis of mathematical structure, in P. Kahn and J. Kyle (eds.), Effective Learning and Teaching in Mathematics and its Applications, Kogan Page Ltd., London, 2002, 20-33.

[4] R.P. Dahlberg and D.L. Housman, Facilitating learning events through example generation, Educational Studies in Mathematics, 33 (1997), 283-299.

[5] O. Hazzan and R. Zazkis, Constructing knowledge by constructing examples for mathematical concepts, Proceedings of the 21st International Conference for the Psychology of Mathematics Education, Vol. 4 (1997), 299-306.

[6] R.C. Moore, Making the transition to formal proof, Educational Studies in Mathematics, 27 (1994), 249-266.

[7] A.H. Schoenfeld, Purposes and methods of research in mathematics education, in D. Holton (ed.), The Teaching and Learning of Mathematics at University Level - An ICMI Study, Kluwer Acad. Publ., Dordrecht, 2001, 221-236.

[8] A. Selden and J. Selden, The role of examples in learning mathematics, http://www.maa.org/t_and_l/sampler/rs_5.html.

[9] D. Tall, The psychology of advanced mathematical thinking, in D. Tall (ed.), Advanced Mathematical Thinking, Mathematics Education Library, Kluwer Acad. Publ., Dordrecht, 1991, 3-21.

[10] D. Tall and S. Vinner, Concept image and concept definition in mathematics with particular reference to limits and continuity, Educational Studies in Mathematics, 12 (1981), 151-169. 
[11] S. Vinner, The role of definitions in the teaching and learning of mathematics, in D. Tall (ed.), Advanced Mathematical Thinking, Mathematics Education Library, Kluwer Acad. Publ., Dordrecht, 1991, 65-81.

\author{
Maria Meehan, \\ Department of Mathematics, \\ University College Dublin, \\ Belfield, Dublin 4 \\ maria.meehan@ucd.ie
}

Received on 20 November 2002. 